Monográfico: Reflujo Vesicoureteral

Arch. Esp. Urol., 61, 2 (341-348), 2008

\title{
REFLUJO VESICOURETERAL EN EL ADULTO
}

\author{
David García Ortells, Fernando González-Chamorro, Eldiberto Fernández Fernández y Andrés \\ De Palacio España.
}

Servicio de Urología Adultos. Hospital San Rafael de Madrid. Madrid. España.

\begin{abstract}
Resumen.- El reflujo vesicoureteral primario se define como la disfunción de la unión vesicoureteral en ausencia de otra patología vesical subyacente. La mayoría de la literatura aborda el reflujo vesicoureteral en la infancia, prestando poca atención al reflujo del adulto. Poco se sabe sobre la incidencia real de esta patología en adultos y poco se ha publicado sobre en que situaciones debemos sospechar, buscar y tratar un reflujo en adultos. En este capítulo se ha hecho una revisión de la literatura sobre cuestiones tan importantes como la epidemiología, diagnóstico y tratamiento del reflujo en adultos. El objetivo de este artículo es trasmitir ante que manifestaciones clínicas debemos buscar un reflujo del adulto y si este es diagnosticada cuando debe ser tratado.
\end{abstract}

Palabras clave: Reflujo vesicoureteral. Adulto. Sustancias inyectables.

Summary.- Primary vesicoureteral reflux is defined as the dysfunction of the vesicoureteral junction in the absence of any other bladder pathology. Most works in the literature focus on pediatric vesicoureteral reflux, paying little attention to reflux in adults. There is not much knowledge about the real incidence of this pathology in adults and there are few published papers about what are the situations in which we should suspect, perform a diagnostic work up and treat reflux in adult patients. It is article we perform a bibliographic review on topics as important as epidemiology, diagnosis and treatment of reflux in adults. The objective of this article is to transmit what are the clinical manifestations in front of which we should look for adult reflux and when to treat when it is diagnosed.

Keywords: Vesicoureteral reflux. Adults. Injectable substances.

\section{INTRODUCCIÓN}

El reflujo vesicoureteral primario, es decir el paso de orina contracorriente de la vejiga al ureter, se define como una disfunción de la unión vesicoureteral en ausencia de cualquier otra patología vesical subyacente como vejiga neurógena, obstrucción infravesical, neoplasia vesical o cirugía previa. El reflujo vesicoureteral resulta de un defecto de la maduración del mesodermo mesonéfrico aunque la razón es desconocida y el mecanismo controvertido.

La mayoría de la literatura urológica aborda el reflujo vesicoureteral en niños y el vesicoureteral en adultos apenas ha recibido la atención que merece. Esto ha provocado que la incidencia de esta patología sea muy poco conocida y variable en cada estudio. Parece ser que el ma- 
yor uso de la cistouretrografía miccional para el estudio del aparato urinario del adulto ha tenido como consecuencia un aparente aumento en la incidencia del reflujo vesicoureteral del adulto (1-3)

La tendencia del reflujo vesicoureteral a mejorar o cesar con el tiempo es algo limitado a la infancia (1). En el adulto, el reflujo normalmente persiste en el tiempo. A pesar que tanto tratamientos médicos como quirúrgicos se aplican en adultos, el manejo del reflujo en esta época de la vida permanece controvertido. La corrección quirúrgica solo debe ser considerada en los casos en los que el riñón mantenga su función conservada y la vía urinaria presente buena tonicidad. Algunos piensan que el tratamiento quirúrgico siempre está indicado, mientras que otros no pueden encontrar ninguna ventaja en el manejo quirúrgico frente al manejo médico.

En una revisión de la literatura reciente, es llamativa la poca atención que ha despertado la prevalencia, sintomatología y fisiopatología del reflujo vesico-ureteral, ya que los artículos de más actuales prácticamente solo se centran en el tratamiento endoscópico, las diferentes sustancias inyectadas y las diferencias en resultados entre ellas.

\section{EPIDEMIOLOGÍA}

La historia natural del reflujo en el niño, a diferencia del adulto, es la tendencia a la desaparición espontánea conforme el niño va creciendo. En 1966 Baker y cols. sugirieron un porcentaje de remisiones espontáneas de un $80 \%$ (4). Esta tendencia a la desaparición se cree que es debido al alargamiento del uréter intravesical y al desarrollo de la musculatura de la unión ureterovesical (2).

La existencia de reflujo vesicoureteral en adultos ha sido registrada en la literatura aunque no existen muchos datos sobre su incidencia. Lipsky y Chisholm sugirieron en 1971 que la incidencia del reflujo en adultos podría ser más alta que la comunicada en la literatura (3). Autores como Valadka y cols. en 1960 (5), Dodge en 1963 (6) y Ross en 1965 (7) informaron de incidencias que variaban entre el 4 y el $25 \%$. Sin embargo, otros como Estes y Brooks en 1970 después de estudiar 903 cistografías en adultos encontraron que el $5 \%$ de estas presentaban reflujo (8). En 1977 Viville y cols. en su publicación mencionaron que Murnaghan en 1965 encontró un $8 \%$ de reflujo en adultos que habían presentado infecciones urinarias (9). Para Baker, en 1966, este porcentaje fue del 5,2\% en 210 adultos explorados (4). En 1990 Chapple, estudiando 519 cistografías realizadas en hombres encontró un 5,1\% de reflujos primitivos (10). En una publicación reciente del año 2005, se estudiaron con cistografía a 86 mujeres jóvenes que habían presentado pielonefritis no complicadas. Sólo 2 de ellas presentaron reflujo vesicoureteral de entra las 86 mujeres, lo que supone un $2,3 \%$ (11).

Un estudio realizado por Scott en 1977 encontró una frecuencia equivalente entre los dos sexos, aunque la mayoría de estudios constatan una preponderancia por el sexo femenino con una relación $3: 1$, predominancia más acentuada en el reflujo primario (12). Clásicamente el reflujo primitivo que aparece en adultos, se suele presentar entre la segunda y tercera decena (13), sin embargo en el varón la frecuencia aumenta a partir de los 60 y 70 años a expensas de reflujo secundario por otras patologías asociadas.

La insuficiencia renal terminal por nefropatia por reflujo parece más frecuente en la mujer. Según Köhler este hecho, sería en parte debido a que las mujeres presentan más fácilmente infecciones urinarias en comparación con el varón facilitando el diagnóstico de reflujo (14). En cambio, la nefropatía por reflujo es más severa en el varón asociada más frecuentemente a lesiones renales bilaterales, posiblemente debido a la menor sintomatología en el varón (13).

\section{MANIFESTACIONES CLÍNICAS}

Las manifestaciones clínicas son numerosas, variadas y sin que exista ninguna particular. Si en la infancia el reflujo está generalmente asociado a infección, en el adulto no ocurre lo mismo, por lo que el reflujo es, en general, mejor tolerado en la edad adulta (15). El reflujo no infectado del adulto desemboca excepcionalmente en una afectación renal. Puede llevar a provocar una leve dilatación de la vía urinaria pero es excepcional encontrarnos con una atrofia renal. Cuando aparecen los síntomas que revelan el reflujo están casi siempre ligados a la infección, lo que conlleva que los paciente con reflujo vesicoureteral se presenten con infecciones urinarias de repetición. Particularmente en la edad adulta, otras manifestaciones pueden aparecer dificultando el diagnóstico y contribuyendo a un retraso en el diagnóstico de 1 a 30 años (13). Los manifestaciones clínicas reveladores del reflujo descritas por diferentes autores quedan reflejadas en la Tabla I.

Aunque el reflujo vesicoureteral en sus formas más leves puede ser asintomático y bien tolerado, de un 50 a un $70 \%$ de los reflujos aparecen como cistitis recidivantes o pielonefritis de repetición (13). Dolores lumbares inespecíficos, proteinuria, atrofia renal unilateral, hipertensión arterial, bacteriuria asintomática o complicación de un embarazo son factores que nos deben hacer sospechar. Es frecuente encontrar la asociación de un reflujo vesicoureteral y una litiasis renal. Hallazgo claramente más asociado al reflujo del adulto que del niño y nada fortuito como demuestran los hallazgos de Torres (27) (asociación en un $18 \%$ de los casos) o Köhler (14).

El mérito de haber señalado la asociación entre reflujo y pielonefritis en el adulto se la debemos a Hodson y Edwards en 1960 (28). Si sobre la existencia de reflujo en el adulto no existe ninguna duda, el mecanismo de lesión renal no está aclarado. Crevy (29) y Hodson estimaron que el reflujo puede provocar una atrofia parenquimatosa como consecuencia de las presiones elevadas que este somete al riñón. Una revisión de la literatura parece, al contrario, confirmar que el reflujo en si no es dañino para el riñón, sino que su mecanismo de lesión reside en la infección $(15,30)$. La formación de lesiones renales tendrá lugar vía ascendente, donde el paso del gérmenes al parénqui- 
ma renal se producirá por medio del reflujo pielotubular. El reflujo y la infección constituyen una entidad clínica en la que el elemento esencial para la lesión renal reside en la infección.

El papel del reflujo en la génesis de la atrofia renal parece posible. La frecuencia de esta asociación varía entre el $40 \%$ y el $90 \%$ según los autores (15). Las observaciones de Heritier en 1975 excluyeron el origen disembrioplástico de la atrofia renal en pacientes con reflujo (15). Parece que el reflujo por si mismo no es capaz de generar una atrofia renal, sino más bien sería la infección urinaria asociada.

Zhang y Baley en 1995 encontraron que tan solo en el 5,2\% de los casos aparecía el clásico dolor lumbar miccional con la vejiga llena (26). La hipertensión arterial aparece de un 1,5 a un $34 \%$ de los casos y se presenta más frecuentemente asociada a la presencia de cicatrices renales bilaterales que unilaterales $(14,21)$ y sobretodo a la presencia de proteinuria e insuficiencia renal. La proteinuria, según Köhler, es el segundo signo revelador de nefropatía por reflujo (14). En la serie de este autor la proteinuria apareció en un $14 \%$ de los casos, cifra similar a las halladas por Malek (19) en el $16 \%$ y Barbe (23) en el $18 \%$ de los casos. La proteinuria es considerada como un excelente marcador pronóstico de la evolución futura de la función renal.

Las manifestaciones clínicas constituyen un elemento nada despreciable, sobre todo a nivel de la conducta terapéutica que no debe estar basada únicamente en los criterios radiológicos y biológicos.

\section{EVOLUCIÓN}

La evolución natural del reflujo vesicoureteral es tal que una vez constituidas las lesiones renales parenquimatosas no solo no regresan, sino que evolucionan por su propia cuenta. Esta evolución es debida a los cambios vasculares y circulatorios secundarios a la hiperfiltración de los glomerulos restantes más que nuevas lesiones infecciosas. Alcanzado este punto, la corrección quirúrgica del reflujo no tendrá ninguna influencia sobre esta evolución. La cirugía antirreflujo no está indicada si el objetivo de la misma es detener el empeoramiento de la función renal.

La presencia o ausencia de proteinuria parece ser un excelente marcador pronóstico. Proteinuria $>1.5 \mathrm{gr}$. cada 24 horas, en especial con cicatrices renales y empeoramiento de la función renal, es indicativo de una progre-

TABLA I. MANIFESTACIONES CLIINICAS DEL REFLUJO VESICOURETERAL SEGÚN SERIES DE DIFERENTES AUTORES.

\begin{tabular}{|c|c|c|c|c|c|c|}
\hline AUTOR & AÑO & DOLOR LUMBAR & INFECCIÓN URINARIA & PNA & HTA & PROTEINURIA \\
\hline KONTTORI (16) & 1977 & $66 \%$ & $85 \%$ & $48 \%$ & - & $18,5 \%$ \\
\hline AUBERT (17) & 1978 & $63 \%$ & $59 \%$ & $50 \%$ & $16,6 \%$ & - \\
\hline DOUNIS (2) & 1978 & $72 \%$ & $72 \%$ & $9 \%$ & $13 \%$ & - \\
\hline ERICHSEN (18) & 1980 & - & $89 \%$ & $50 \%$ & $5,4 \%$ & $4 \%$ \\
\hline MALEK (19) & 1983 & $16,5 \%$ & - & - & $34 \%$ & $16,4 \%$ \\
\hline NEVES (20) & 1983 & - & $70 \%$ & - & $24 \%$ & - \\
\hline REGIN (21) & 1984 & $9 \%$ & $69 \%$ & $46 \%$ & $14 \%$ & $7 \%$ \\
\hline HERMANOWITZ (22) & 1984 & $7,7 \%$ & $12,8 \%$ & $25,6 \%$ & $5 \%$ & - \\
\hline BARBE (23) & 1984 & $33 \%$ & $45 \%$ & $57 \%$ & $18 \%$ & $18 \%$ \\
\hline CHOQUENET (24) & 1984 & $10,8 \%$ & $78,3 \%$ & $70,2 \%$ & - & $5,4 \%$ \\
\hline LI (25) & 1985 & $63,1 \%$ & $47,6 \%$ & $67 \%$ & $10,6 \%$ & - \\
\hline ZHANG-BAILEY (26) & 1995 & $5,1 \%$ & - & - & $8,5 \%$ & $4,8 \%$ \\
\hline
\end{tabular}


siva nefropatía por reflujo (19). En estas circunstancias, la cirugía antirreflujo raramente está indicada al menos que aparezcan pielonefritis sintomáticas recurrentes e incontrolables.

\section{DIAGNÓSTICO}

Respecto al diagnóstico por imagen del reflujo vesicoureteral en adultos, técnicamente no plantea ningún problema en particular con respecto al de la infancia. El hecho de encontrar, en el aparato urinario superior, restos de contraste introducido en la vejiga en el transcurso de una cistografía retrógrada traduce la existencia de un reflujo vesicoureteral. En la forma clásica de diagnóstico de reflujo, la cistogrrafía retrógrada y miccional continua siendo el examen de referencia para establecer el diagnóstico y grado de relujo vesicoureteral (Figura 1). La única diferencia digna de mención es la cistografía isotópica, técnica con una sensibilidad superior a la cistografía radiológica y técnicamente más fácil de realizar en el adulto que en el niño (Figura 2). En 2007, McMahon ha publicado un artículo sobre el uso de la resonancia magnética en el diagnóstico del reflujo (31). La RNM obtuvo mayor precisión diagnóstica que la ecografía a nivel de la información estructural renal y resultados comparables a la pruebas de medicina nuclear en la estimación de la función renal.

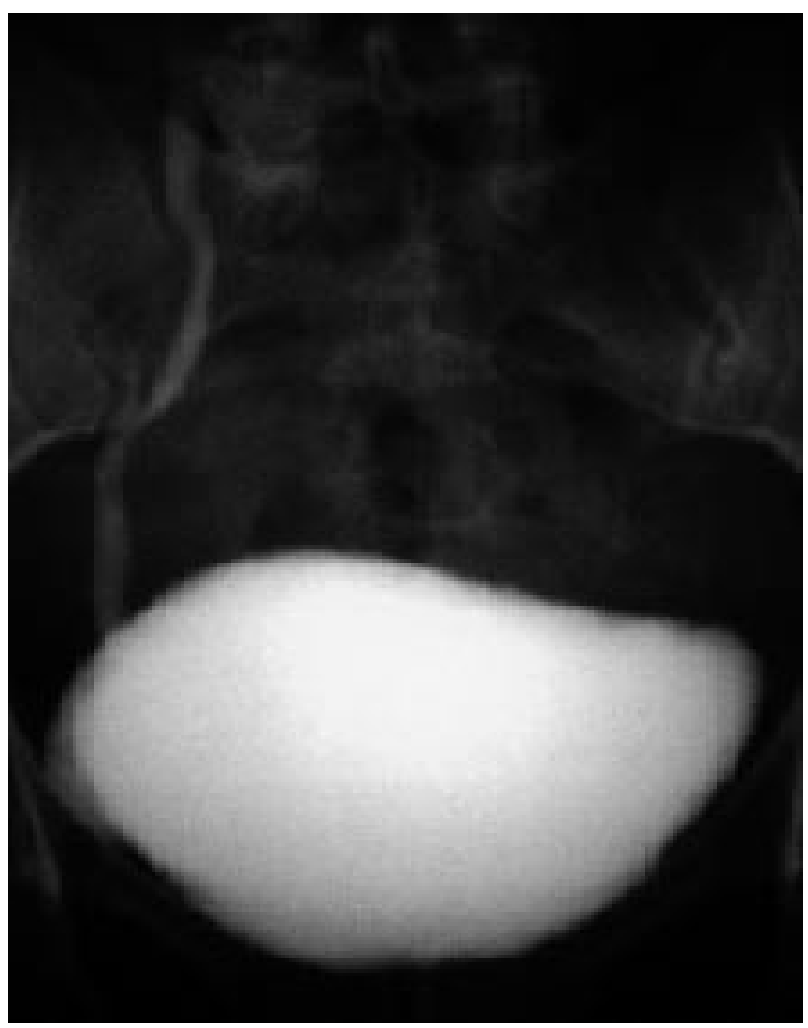

FIGURA 1. Reflujo vesicoureteral objetivado mediante cistografía.
En este apartado se plantea y se centra en la cuestión de cuando buscar un reflujo vesicoureteral en adultos. Ante esta pregunta debemos plantearnos primero la rentabilidad de las pruebas para descubrir un reflujo y segundo las consecuencias terapéuticas del descubrimiento de un reflujo. Es evidente que si el diagnóstico de un reflujo no conlleva una actitud terapéutica, la simple curiosidad diagnóstica no justifica la búsqueda sistemática de esta patología. Como se comentó en el apartado sobre la epidemiología, la prevalencia del reflujo, si bien varía en función de las series, no parece ser muy superior al 5\%. Este porcentaje tan bajo es una primera respuesta a la pregunta de la rentabilidad insuficiente de la realización sistemática de la cistografía. David y cols. encontraron que a partir de los 9 años la proporción de reflujo disminuye significativamente hasta cifras similares a las del adulto, por lo que no recomienda la realización de cistografía sistemáticas a partir de esta edad (32).

En cambio si realizamos cistografías en pacientes que padecen proteinuria o cicatrices renales, la proporción de reflujos diagnosticados puede ser bastante más importante. Según Moreau y cols. encontraríamos hasta un $2 / 3$ de casos, de manera que aumentaríamos de forma importante la rentabilidad de la cistografía (33).

Los resultados tan desalentadores que se obtienen en la corrección quirúrgica del reflujo en pacientes que padecen cistitis de repetición, no motiva la búsqueda de reflujo vesicoureteral en estas situaciones (34).

Ante el primer episodio de pielonefritis aguda, se aconseja la realización de al menos una ecografía renal, no siendo necesario la práctica de una cistografía (35). Ante pielonefritis agudas recidivantes frecuentes en el adulto joven y en particular en la mujer en edad fértil, la búsqueda de reflujo está justificada ya que su diagnóstico puede desembocar en un acto terapéutico.

En el reflujo del adulto, merece atención especial el papel de la cistoscopia. Ambrose (36) en 1945, Williams en 1971 (37) constataron que en la mayoría de casos de pielonefritis asociadas a reflujo encontraron anomalías de posición del orificio ureteral. Estas anomalías en los meatos ureterales observadas en cistoscopia representan el sustrato anatómico de una displasia del uréter intramural reponsable del reflujo. Estos defectos de posición son muy frecuentemente encontrados en el adulto con reflujo vesicoureteral asociado a pielonefritis crónica o atrofia renal homolateral.

\section{TRATAMIENTO}

A la hora de establecer una estrategia en el tratamiento del reflujo vesicoureteral primario hay que tener en cuenta los diferentes hallazgos clínicos, analíticos así como los resultados de las pruebas de imagen. En caso de reflujo secundario el tratamiento estará dirigido al factor etiológico. El objetivo del tratamiento es mantener la función renal, la prevención de las progresivas cicatrices renales, la hipertensión y evitar la morbilidad asociada a las infecciones de repetición del tracto urinario alto. 
El tratamiento del reflujo vesicoureteral es a la vez médico y quirúrgico. Por un lado combate la infección urinaria y por otro suprime el reflujo al riñón el cual es el que perpetua la infección y el daño renal. Respecto al tratamiento médico, si bien la antibioterápia es indiscutible durante los episodios infecciosos agudos, el tratamiento antibiótico preventivo a largo plazo de los procesos infecciosos es difícilmente concebible en el adulto por su poca eficacia (20). Desde 1977, Senoh no solo demostró que el tratamiento profiláctico antibiótico prevenía mal las infecciones sino también la alta tasa de efectos de intolerancia al tratamiento (38). Donde la antibioterapia tiene sentido y donde muchos autores coinciden, es el interés de mantener un tratamiento antibiótico de forma prolongada en el postoperatorio $(39,34)$. Antes de 1975 las técnicas quirúrgicas más comúnmente utilizadas eran las del Politano-Lead-Better o Glenn-Anderson. Después de esta fecha las técnicas de avance submucoso como la de Cohen paso a ser las más utilizadas si bien la disección ureteral endovesical es más difícil que en el niño. En la Tabla II quedan reflejados los resultados de estas técnicas quirúrgicas entre las más importantes series de tratamiento del reflujo vesicoureteral en adultos.

La simplicidad e inocuidad del tratamiento endoscópico ha provocado su amplia aceptación en el reflujo del adulto manifiestamente con menor reticencia que en el niño, con resultados comparables a la cirugía tradicional.
En las series de Salas-Sironvalle y Dessouki ambos obtuvieron un $86 \%$ de buenos resultados $(42,43)$. El tratamiento endoscópico (Figura 3) no solo ha demostrado su eficacia como tratamiento primario del reflujo sino también tras el fracaso de las cirugías de reimplantación ureteral tal y como comunicaron Cloix y Kumar en 1992 (44,45). Este autor llegó a obtener resultados de hasta 94,5\% de éxito después de tres inyecciones endoscópicas. Durante los primeros años del nuevo siglo se publicaron otros estudios donde se revisaban los resultados del tratamiento endoscópico así como las diferencias entre ellos. En 2004 Choo y cols. comunicaron su experiencia sobre 34 uréteres, los cuales fueron tratados con polidimetilsiloxano (Teflon®). En función del reflujo grado I, II, III y IV, obtuvieron unas tasas de éxito del $90 \%, 87,5 \%, 79,9 \%$ y $100 \%$ respectivamente (46). Ese mismo año, Sugiyama y cols. publicaron los diferentes resultados obtenidos en la cirugía endoscópica con diferentes sustancias. Sus porcentajes de éxito fueron $8 \%$ para inyecciones con grasa autóloga, $53 \%$ con polimeros de hialurónico/dextranómero (Deflux®), 63\% con PTFE y $56 \%$ con GAX (colágeno). Entre sus conclusiones destacaron que la grasa autóloga no tiene utilidad en la práctica clínica por su baja durabilidad y que PTFE no es una sustancia cuya seguridad esté todavía establecida. A pesar de las ventajas de menor invasividad y posibilidad de re-inyección de las técnicas endoscópicas, las tasas globales de éxito con colágeno y Deflux $®$ fueron insuficientes comparadas con la cirugía de reimplantación ureteral (47).

TABLA II. RESULTADOS SEGÚN DIFERENTES TÉCNICAS QUIRÚRGICAS.

\begin{tabular}{|c|c|c|c|c|}
\hline AUTOR & TÉCNICA & BUENOS RESULTADOS & ESTENOSIS & REFLUJO RESIDUAL \\
\hline AUBERT-DORE (17) & $29 \mathrm{C}-3 \mathrm{PL}$ & $96,2 \%$ & 0 & $3,8 \%$ \\
\hline DE SY (40) & $41 \mathrm{GA}-31 \mathrm{~K}$ & $100 \%$ & 0 & 0 \\
\hline DAVIN-FAURE (41) & $63 \mathrm{C}-3 \mathrm{PL}$ & $95,2 \%$ & 0 & $4,8 \%$ \\
\hline ERICHSEN (18) & $116 \mathrm{PL}$ & $86 \%$ & $9 \%$ & $5,2 \%$ \\
\hline REGIN-BITTARD (21) & $25 \mathrm{LG}$ & $74 \%$ & $8,5 \%$ & $17,5 \%$ \\
\hline BARBE-LOBEL (23) & $57 \mathrm{C}$ & $91,3 \%$ & $5,3 \%$ & $8,7 \%$ \\
\hline LI-CUKIER (25) & $103 \mathrm{C}$ & $93 \%$ & $1,3 \%$ & $5,7 \%$ \\
\hline SALAS (42) & Endoscópica & $86 \%$ & - & - \\
\hline DESSOUKI (43) & Endoscópica & $86 \%$ & - & - \\
\hline LAVELLE (49) & Endoscópica & $84-73 \%$ & - & - \\
\hline CHOO (46) & Endoscópica & $86 \%$ & - & - \\
\hline
\end{tabular}


A finales de 2004, Perez-Brayfield (48) obtuvo algo más de éxito con las inyecciones submucosas de Deflux $\circledast(68 \%)$. Lavelle y cols. en 2005 obtuvieron mejores respuestas con la misma sustancia. En función de los grados I, II, III y IV, sus porcentajes de éxito fueron del $82 \%, 84 \%, 78 \%$ y $73 \%$ respectivamente (49). A pesar de su bajo número de pacientes, Okene y cols. en 2006, trataron a 9 pacientes portadores de reflujo con corrección endoscópica. A los 3 meses la cistografía miccional demostró la desaparición del reflujo en 8 de ellos (50).

El hecho que el reflujo vesicoureteral en el adulto es a menudo bien tolerado, que los resultados quirúrgicos no son tan buenos como en el niño y que el riesgo de estenosis en la cirugía tradicional es mayor que en la infancia, hace que el reflujo en adultos sea menos quirúrgico que en el niño. A partir de los 40-50 años la corrección quirúrgica del reflujo no tiene mucha justificación siendo, a partir de esta edad, el tratamiento endoscópico una solución interesante (13).

Las indicaciones quirúrgicas del reflujo en el adulto son más fáciles de establecer que en el niño. Un adulto que presenta un reflujo asintomático asociado o no a una bacteriuria asintomática no requiere más que un seguimiento clínico y analítico controlando la tensión arterial, la pro-

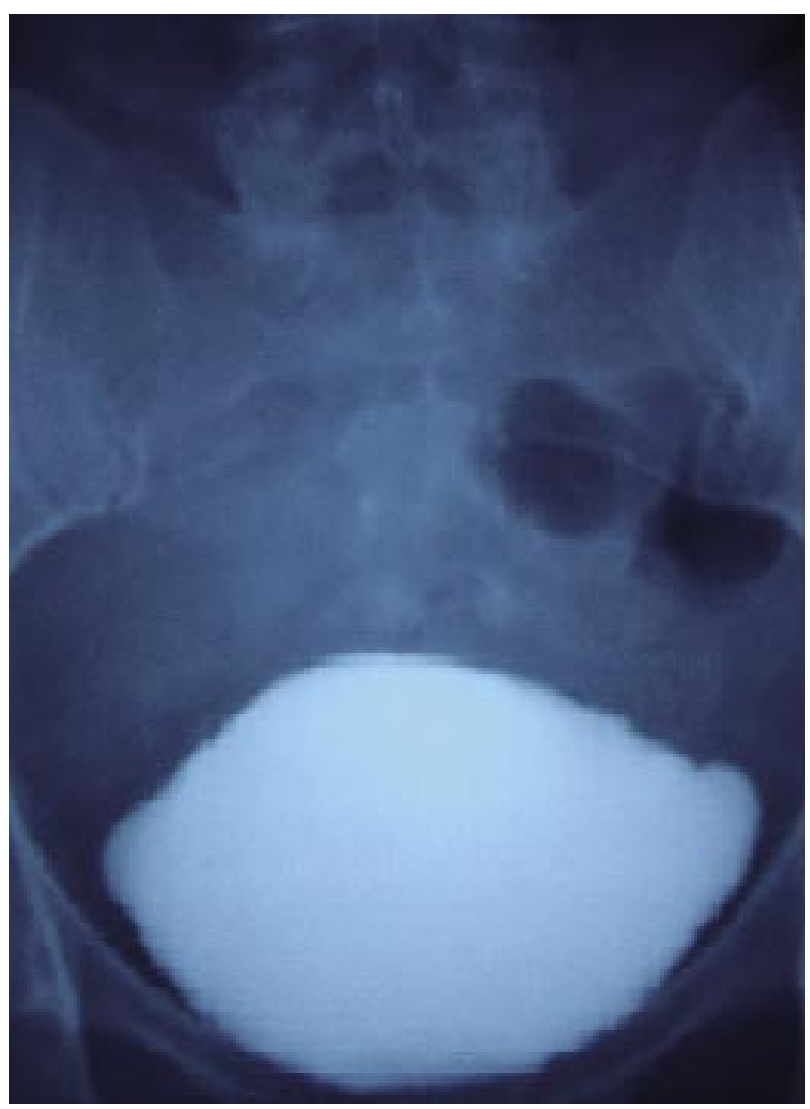

FIGURA 2. Reflujo vesicoureteral corregido tras cirugía. teinuria y la función renal. El hecho de aparición de estos factores implica la necesidad de valoración nefrológica. El reflujo secundario con infección urinaria recidivante es raro y la frecuencia no sobrepasa el $4 \%$ (15). Este reflujo es a menudo transitorio, a penas representa riesgo para el riñón y suele desaparecer después de un tratamiento médico apropiado. La mujer en edad fértil supone una situación especial en la que la presencia de una bacteriuria asintomática aumenta el riesgo de infección urinaria gestacional y justifica la profilaxis antibiótica desde el comienzo del embarazo (13).

Si por el contrario nos encontramos con un reflujo sintomático sobre un riñón funcional, una corrección quirúrgica del reflujo estaría indicada sobre todo en mujeres en edad fértil. Si la cirugía está indicada es preferible que se lleve a cabo cuando la vejiga esté libre de infección y edema. En el caso de que el reflujo fuera unilateral, no sería necesario la reimplantación sistemática del uréter contralateral debido a la dificultad técnica y al riesgo de aparición de complicaciones. De todas formas, el orificio contralateral debe ser valorado preoperatoriamente. Si el paciente presenta una historia de reflujo bilateral, el orificio contralateral presenta una anomalía de posición y el riñón homolateral presenta áreas de cicatrices en el parénqui$\mathrm{ma}$, una cirugía de antirreflujo bilateral debe ser tenida en cuenta.

En el caso de reflujo en el contexto de una duplicidad ureteral, se puede aplicar el mismo razonamiento. De todas formas habría que reseñar que en este tipo de malformación, las lesiones renales y ureterales son tales que la cirugía conservadora no está a menudo justificada. La heminefrectomía es, frecuentemente, el único tratamiento posible y razonable. Destacar la importancia de asociar la ureterectomía para evitar dejar un muñón ureteral que perpetúe la infección urinaria (3).

Ante un reflujo sintomático sobre un riñón atrófico podría estar justificada la nefrectomía. El hallazgo de

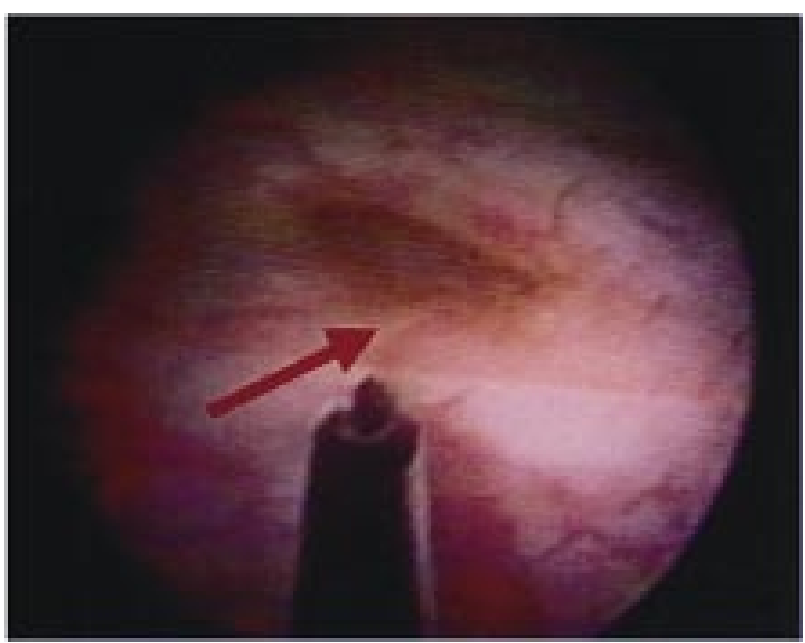

FIGURA 3. Aspecto endoscópico durante la corrección del reflujo mediante inyección de sustancias. 
un riñón pequeño no funcional asociado a un reflujo asintomático no implicaría la necesidad de una nefrectomía sistemática ya que a pesar del tratamiento médico o quirúrgico, la proporción de adultos que desembocarán en una insuficiencia terminal por nefropatía de reflujo permanece inalterable. La reimplantación ureteral está contraindicada cuando el reflujo presenta una alteración radiológica grave con dilatación de la vía urinaria y alteración marcada de la función renal. Llegado a este estado, el riñón evoluciona a una nefropatía independientemente de una intervención. Como se comentó anteriormente una proteinuria > 1,5 gr. en 24 horas, especialmente asociada a empeoramiento de la función renal y cicatrices renales, es indicativo de nefropatía por reflujo. En estas circunstancias la cirugía antirreflujo raramente está justificada. Haubensack demostró que con una creatinina superior a $2.3 \mathrm{mg}$ la corrección quirúrgica del reflujo podría ser más nociva que beneficiosa (15).

Después de la corrección del reflujo, si los resultados obtenidos han sido satisfactorios, el seguimiento se puede realizar con control ecográfico. La realización de una cistografía no está justificada a menos que se tengan dudas del resultado obtenido en la cirugía.

Con respecto a los resultados quirúrgicos, la mayoría de las series obtienen unos porcentajes de supresión de reflujo muy elevados (Tabla II). La persistencia de reflujo tras la cirugía es muy rara, siendo la verdadera complicación la estenosis del uréter reimplantado, igualmente excepcional (Tabla II), que puede comprometer la función renal. La eficacia de la cirugía para la desinfección urinaria está menos asegurada siendo las tasas de curación de la infección entorno 60 al $70 \%$ (51). Esto conlleva que la cistitis puede llegar a ser un problema ocasional persistiendo después de la cirugía.

\section{CONCLUSIONES}

- El reflujo del adulto es más frecuente de lo comúnmente admitido, a menudo bien tolerado y con poca tendencia a la remisión espontánea a diferencia del reflujo en la infancia.

- El reflujo primitivo de la mujer es alrededor 3 veces más frecuente que en el hombre.

- En el adulto, el mecanismo de lesión renal reside en la infección urinaria.

- Ante la presencia de un reflujo asintomático sin repercusión sobre el riñón, el simple seguimiento clínico y analítico puede ser suficiente.

- La cirugía de corrección del reflujo no se debe realizar a menos que el riñón mantenga una buena función renal y la vía urinaria una buena tonicidad. El tratamiento quirúrgico está contraindicada si existe una importante afectación renal con dilatación y atonía del uréter.

- La simplicidad, inocuidad, posibilidad de re-inyección y sus buenos resultados ante el fracaso del reimplante urete- ral, hacen que el tratamiento endoscópico sea, probablemente, en la actualidad la primera actitud quirúrgica en el adulto.

\section{BIBLIOGRAFÍA y LECTURAS RECOMENDADAS ( lectura de interés $y$ ** lectura fundamental)}

1. NATIV, O.; HERTZ, M.; HANANI, Y. y cols.: "Vesicoureteral reflux in adults: A review of 95 patients". Eur. Urol., 13: 229, 1987.

2. DOUNIS, A.; DUNN, M.; SMITH, P.J.B.: "Ureteric Reimplantation for vesico-ureteric reflux in adult". Br. J. Urol., 50: 233, 1978.

*3. LIPSKY, H.; CHISHOLM, G.D.: "Primary vesicoureteric reflux in adults". BJU, 43: 227, 1971.

4. BAKER, R.; MAXTED, W.; MAYLATH, J. y cols.: "Relation of age, sex and infection to reflux". J. Urol., 95: 27,1966.

5. VALADKA, B.; COTTRELL, T.; LLOYD, F.A.: "Significance of vesico-ureteral reflux. Analysis of cystograms in paraplegics". Am. J. Surg., 100: 527, 1960.

6. DODGE, E.A.: "Vesico-ureteric reflux: Diagnosis with iodine-131 sodium ortho-iodohippurate". Lancet, 1: 303, 1963.

7. ROSS, J.C.: "Vesico-ureteric reflux in the neurogenic bladder". British Journal of Surgery, 52: 164, 1965.

*8. ESTES, R.C.; BROOKS, R.T.: "Vesico-ureteral reflux in adults". J. Urol., 103, 1970.

*9. VIVILLE, C.: "Le reflux vésico-rénal primitif de l'adulte". J. Urol. Nephrol., 83: 481, 1977.

10. CHAPPLE, C.R.; CHRISTMAS, T.J.; TURNERWARWICK, R.T.: "Vesicoureteric reflux in the adult male". Br. J. Urol., 65: 144, 1990.

11. CHOI, Y.D.; YANG, W.D.; DO, S.H. y cols.: "Vesicoureteral reflux in adult women with uncomplicated acute pyelonephritis". Urology, 66: 55, 2005.

12. SCOTT, J.E.S.: "The management of ureteric reflux in children". Br. J. Urol., 49: 109, 1977.

**13. No authors listed. "Reflux Primitif de l'adulte". Prog. Urol., 8: 923, 1998.

*14. KÖHLER, J.; TENCER, J.; THYSELL, H. y cols. "Vesicoureteral reflux diagnosed in adulthood. Incidence of urinary tract infections, hypertension, proteinuria, backpain and renal calculi”. Nephrol. Dial. Transplant., 12: 2580,1977

*15. HERITIER, P.; ZINGG, E.: "Vesico-renal reflux in adults". J. Urol. Nehrol. (Paris), 81: 497, 1975.

16. KONTTURI, M.J.; KOSKELA, M.A.: “Active surgical management of primary vesicoureteral reflux in adults". Scand. J. Urol. Nephrol., 11: 239, 1977.

17. AUBERT, J.; DORE, B.; DENIS, P. y cols.: "La tolérance à long terme du reflux vésico-rénal primitif". J. Urol. Nephrol., 3: 195, 1978.

18. ERICHSEN, C.; GENSTER, H.G.: "Vesico-ureteral reflux in non-paediatric patients". Scand. J. Urol. Nephrol., 14: 233, 1980.

*19. MALEK, R.S.; SVENSSON, J.; NEVES, R.J. y cols.: "Vesicoureteral reflux in the adult. III. Surgical correction: Risks and benefits”. J. Urol., 130: 882, 1983. 
*20. NEVES, R.J.; TORRES, V.E.; MALEK, R.S. y cols.: "Vesicoureteral reflux in the adult.IV. Medical versus surgical management". J. Urol., 132: 882, 1983.

21. REGIN, J.P.; PELTRE, G.; JUNG, J.L. y cols.: "Traitment du reflux vésico rénal primitif de l'adulte. A propos de 71 observations". Ann. Urol., 18: 125, 1984.

22. HERMANOWITZ, P.; DUCASSOU, J.; SERMENT, G. y cols.: "Réflexions sur une série de trent-neuf cas de reflux vésico-rénal de l'adulte”. Ann. Urol., 18: 52, 1984.

23. BARBE, Y.P.; LOBEL, B.: "Reflux vésico-rénal de l'adulte. Etude de cinquante sept uréteres réimplantés par avancement sous-muqueux". Ann. Urol., 18: 49, 1984.

24. CHOQUENET, C.; JULIA, P.; DUFOUR, B.: "Le reflux vésico-rénal découvert chez l'adulte. A propos de 37 observations". Ann. Urol., 18: 59, 1984.

25. LI, Z.; TERDJMAN, S.; BEURTON, D. y cols.: "Le reflux vésico-urétéral de l'adulte. A propos de 103 observations". J. Urol. (Paris), 91: 589, 1985.

26. ZHANG, Y.; BAILEY, R.R.: "A long terme follow up of adults with reflux nephropaty". N.Z. Med., 108: 142, 1995.

*27. TORRES, V.E.; MALEK, R.S.; SVENSSON, J.P.: "Vesicoureteral reflux in adult. II. Nephropaty, hypertension and stones". J. Urol., 130: 41, 1983.

28. HODSON, C.J.; EDWARDS, D.: "Chronic pyelonephritis and vesico-ureteric reflux". Clin. Radiol., 14: 219, 1960.

29. CREEVY, C.D.: "Vesico-ureteral reflux in children. A brief review". Minn. Med., 50: 757, 1967.

30. LENEGHAN, D.; CASS, A.S.: "Long-term effect of vesico-ureteral reflux on the upper urinary tract of boys". J. Urol., 107: 752, 1972.

31. McMAHON, L.P.; KATSOULIS, J.; TROUPIS, J.M. y cols.: "Use of magnetic resonance imaging to assess renal structure and function in reflux nephropaty". Nefrology (Carlton), 12: 172, 2007.

32. DAVID, C.; DACHER, J.N.; MONROC, M. y cols.: "Cystographie rétrograde après un premier épisode de pyélonéphrite aiguë chez la fillette et l'adolescence”. J. Radiol., 79: 133, 1998.

33. MOREAU, J.F.; GRENIER, P.; GRUNFELD, J.P.: "Les atrophies cortico-papillaires segmentaires de l'adulte. Relations avec le reflux vésico-rénal”. J. Urol. Nephrol., 13: 229, 1977.

34. SOCIETE FRANÇAISE D UROLOGIE.: "Réunion du 28 Mai 1983”. Ann. Urol., 18: 46, 1984.

35. ANONYME.: "Cystites et pyélonéphrites aiguës simples de la femme de 15 à 65 ans en dehors de la grossesse. Recommandations et références médicales”. Les guides de 1'Assurance Maladie, 1: 170, 1966.

36. AMBROSE, S.S.: "Reflux pyelonephritis in adults se- condary to congenital lesions of the ureteral orifice". J. Urol., 102: 302, 1969.

37. WILLIAMS, D.F.: "The natural history of reflux". Urol. Int., 26: 350, 1971.

38. SENOH, K.; IWATSUBO, E.; MOMOSES, S. y cols.: "Non-obstructive vesicoureteral reflux in adults: Value of conservative treatment". J. Urol., 117: 566, 1977.

*39. GUTHMAN, D.A.; MALEK, R.S.; NEVES, R.J. y cols.: "Vesicoureteral reflux in the adult. V. Unilateral disease". J. Urol., 146: 21, 1991.

40. DE SY, W.; OOSTERLING, W.; WYNDAELE, J.J.: “A plea for antireflux operations reflux in adults: Review of 50 cases". J. Urol., 120: 549, 1978.

41. DAVIN, J.L.; FAURE, G.; REVOL, M.: "Le traitement chirurgical du reflux vésico-urétéral chez l'adulte. A propos de 45 observations". Ann. Urol., 18: 46, 1984.

42. SALAS-SIROVALLE, M.: "Tratamiento endoscópico del reflujo vesico ureteral congénito". Actas Urol. Esp., 14: $258,1990$.

43. DESSOUKI, T.; STAERMAN, F.; ABBAR, A. y cols.: "Treatment of primaty vesicoureteric reflux by polytetrafluoroethylene injection: A middle term follow up study". Eur. Urol., 23: 375, 1993.

44. CLOIX, P.; DAWHARA, M.; CHOUKAIR, M. y cols.: "Traitment endoscopique du reflux vésico-urétéral après réimplantation de l'uretér (grefes exclues)". Prog. Urol., 2: 66, 1992.

45. KUMAR, R.; PURI, P.: "Endoscopic correction of vesicoureteric reflux in failed reimplanted ureters". Eur. Urol., 2: 66, 1992.

46. CHOO, M.S.; HONG, B.; JI, Y.H. y cols.: "Endoscopic treatment of vesicoureteral reflux with plydimethylsiloxane in adult women". Eur. Urol., 45: 787, 2004.

*47. SUGIYAMA, T.; HANAI, T.; HASHIMOTO, K. y cols.: "Long-term outcome of the endoscopic correction of vesico-ureteric reflux: A comparison of injected substances". BJU Int., 94: 381, 2004.

48. PEREZ-BRAYFIELD, M.; KIRSCH, A.J.; HENSLE, T.W. y cols.: "Endoscopic treatment with dextranomer/ hyaluronic acid for complex cases of vesicoureteral reflux". J. Urol., 172: 1614, 2004.

49. LAVELlE, M.T.; CNLIN, M.J.; SKOOG, S.J.: "Subureteral injection of deflux for correction of reflux: Analysis of factors predincting success". Urology, 65: 564, 2005

50. OKENE, Z.; FROMER, D.; KATZ, M.H. y cols.: "Endoscopic management of vesicoureteral reflux in women presenting with pyelonephritis". J. Urol., 176: 2219, 2006.

51. VIVILLE, C.; DE PETRICONI, R.: "Traitement du reflux vesico-renal primitif chez ládulte. A propos de 41 observations". Ann. Urol. (Paris), 18: 63, 1984. 